Research Paper

\title{
Surgical Treatment for Unstable Distal Clavicle Fracture with Micromova- ble and Anatomical Acromioclavicular Plate
}

\author{
Qingjun Liu*, Jianyun Miao*, Bin Lin ${ }^{\bowtie}$, Kejian Lian \\ Department of Orthopaedic Surgery, the 175th Hospital of PLA (the Affiliated Southeast Hospital of Xiamen University), \\ Orthopaedic Trauma Center of PLA, Zhangzhou, 363000, PR China.
}

${ }^{*}$ Qingjun Liu and Jianyun Miao contributed equally to the work.

Corresponding author: Bin Lin, 268 Zhang-Hua Road, Zhangzhou, 363000, Fujian Province, China. Tel \& Fax: +86-596-2931538; E-mail: miaojianyun@foxmail.com.

(C) Ivyspring International Publisher. This is an open-access article distributed under the terms of the Creative Commons License (http:/ / creativecommons.org/ licenses/by-nc-nd/3.0/). Reproduction is permitted for personal, noncommercial use, provided that the article is in whole, unmodified, and properly cited.

Received: 2012.03.31; Accepted: 2012.06.03; Published: 2012.06.06

\begin{abstract}
Between 2006 and 2009, 18 patients of distal clavicle fracture were treated with micro-movable and anatomical acromioclavicular plate (MAAP) in our department. According to the Neer's classification, all cases were unstable with type IIA (I 2 cases) and type IIB ( 6 cases). Functional outcome was evaluated using the Karlsson's criteria. The mean follow-up was 18 months (range, 12-36months). No postoperative plate screws complication was observed. Osseous union could be achieved at a mean time of 12 weeks after operation in 18 patients (range, 8 -16 weeks). According to Karlsson's criteria, radiographic appearances and postoperative shoulder functional recovery revealed a good and excellent rate in these cases. We conclude that surgical treatment using MAAP seems to be a good option for unstable type II fractures of the distal clavicle. This technique allows for reliable fixation with early functional exercises and functional recovery.
\end{abstract}

Key words: Micro-movable fixation, Distal clavicle, Fracture, Acromioclavicular plate.

\section{Introduction}

Clavicle fractures are relatively common due to their special subcutaneous position. Distal clavicular fracture as a special form of injury have been estimated to account for only $12 \%$ to $15 \%$ of all clavicle fractures ${ }^{[1]}$. According to the fracture pattern and the relationship of the fracture line to the coracoclavicular ligaments and acromioclavicular joint, Neer divided distal clavicular fractures into three types ${ }^{[2-4]}$. In type I fractures, the ligaments are intact with fractures stable. Type III fractures are intra-articular fractures involving acromioclavicular joint surface and are similar to type I fractures which are relatively stable. Type II fractures with ruptured of the coracoclavicular ligaments are less stable than type I and III fractures, which has been subdivided into two types include type IIA and type IIB. Type IIA fractures occur medial to the conoid ligament with coracoclavicular ligaments intact. Type IIB fractures occur between the coracoclavicular ligaments and include disruption of the conoid ligament. The loss of conoid ligament restraint on the lateral fragment could result in instability and a significant displacement of the fractures, and showed a high delayed union or nonunion rate[5]. In addition, for type II fractures, unbalanced forces such as weight of the arm and muscle forces acting on the fracture site are another important factor that hinder union ${ }^{[2]}$. Therefore, surgical methods for treatment of type II fractures are very necessary. 
Although there are several surgical techniques to treat type II fracture of distal clavicle, most of the surgical methods develop to provide stable fixation of fractures across the acromioclavicular joint, which bring about the adverse consequences of limiting the micromovement of the acromioclavicular joint to some extent. To this end, we design a micro-movable and anatomical acromioclavicular plate (MAAP), which not only effectively fixes the unstable distal clavicle fractures, but also retains the micro-movable feature of acromioclavicular joint. The purpose of this study was to report a new surgical technique for treatment of type II unstable distal clavicle fracture with MAAP and retrospectively evaluate the outcome of these patients with clinical follow up.

\section{Materials and methods}

In collaboration with Xiamen Double Engine Medical Material Co Ltd, we have designed a MAAP, for which we have obtained the national invention patent (patent number: 200620009587.3). This plate is made of titanium alloy and was designed in accordance with the surface anatomy of the clavicle and acromion, which is composed by three parts, such as clavicle lateral anatomical plate, acromion medial anatomical plate and micro-movable connecting rods. The plate has 4 screw holes on the acromion side, and clavicle side of 5, 7, or 9 screw holes can be used (Fig. 1).

From February 2006 to July 2009, a consecutive series of 18 patients (10 males and 8 females, 6 left shoulder and 12 right shoulder, aged 17-45 years, with an average age of 32 years) diagnosed with distal clavicle fracture underwent surgery in our department, which were unstable type II fractures according to X-ray examination results and Neer classification, and were included in this study. 10 were injured in traffic accident, 5 were injured in crush injury and 3 in falling injury. 18 cases were fresh fractures with no significant associated injuries. Operations were performed within 7 days (range, 3-14 days) from the original injury in our study. The mean follow-up period was 18 months (range, $12-36$ months).

The surgical procedures were performed under general anesthesia with the patient in the beach-chair position on the operating table by the same surgeon. A length of $6-8 \mathrm{~cm}$ standard Thompson incision was made, centered on the fracture ends. The acromion medial side and proximal clavicle fracture site were fully exposed. The fracture ends were mobilized and given adequate decompression to remove the interposed soft tissue. MAAP was then applied onto the acromion medial side and fracture ends. At least four screws are used to fix the acromion medial side and at least five screws are used to fix the clavicle end which includes two fix to the distal clavicle fracture site and at least three fix to the proximal clavicle fracture site. The torn coracoclavicular ligament should be identified and given the necessary suture and reconstruction by non-absorbable suture. All of the patients were reviewed $X$-rays at three months, six months, one year, two years and three years postoperatively and the shoulder function was evaluated using Karlsson's criteria during the follow-up period[6] (Table 1).

Table I. Karlsson's criteria for assessment of postoperative shoulder functional recovery.

\begin{tabular}{llll}
\hline Category & Degree & & \\
\hline pain & no & subtle & serious \\
myodynamia & normal & medium & weak \\
movement & flexible & $90^{\circ}$ to $180^{\circ}$ & $\begin{array}{l}\text { limited from } \\
\text { every direction }\end{array}$ \\
criteria & A & B & C \\
\hline
\end{tabular}

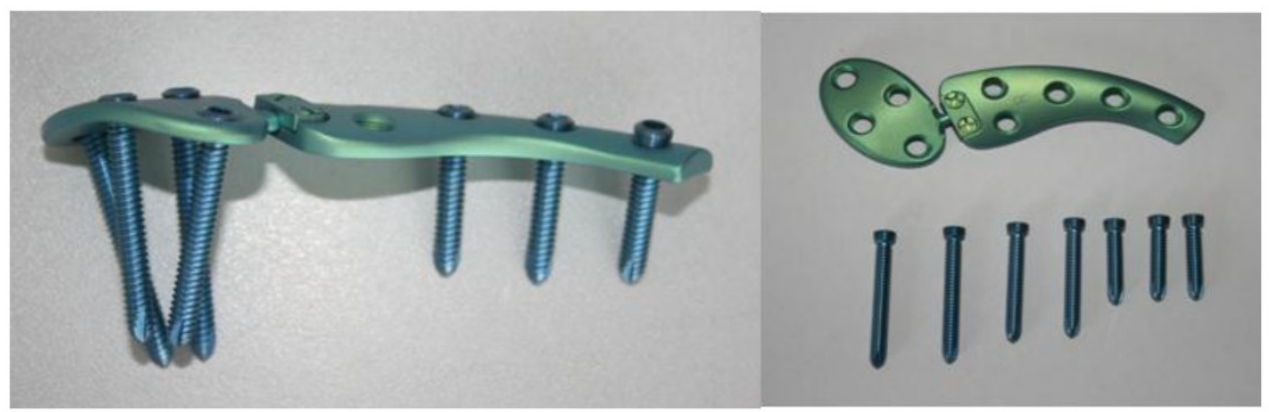

Figure I Pictures for micro-movable and anatomical acromioclavicular plate (MAAP). 


\section{Results}

This group of 18 patients was successfully performed open reduction and internal fixation operation with MAAP. The average operative time was $28 \mathrm{~min}$ (22-38 min). Early passive shoulder joint function exercises were started of temporary immobilisation after 1 week by the patient's family. All patients were instructed examination until the healing of the fracture and followed up for an average of 18 months (range, 12-36months). Fracture fragments of patients showed bony union after 16 weeks through radiologic evaluation (Fig. 2). The mean fracture healing time was 12 weeks after operation (range, 8-16 weeks).At the last follow-up, according to Karlsson's criteria, all patients were assessed good or excellent results: 14 (77.8\%) cases were rated A and 4 (22.2\%) were rated B (Table 2). No complications occurred except for one patient of non-union. We considered that this was probably related to premature internal plate removal postoperatively and performed reoperation using a new plate with a bone graft in the nonunion site promoting fracture healing. The patient ultimately obtained a satisfactory functional result. In addition, there was one patient had a re-fracture at the distal of clavicle as a result of new falling injury which was managed with surgery and also got a satisfactory functional result.

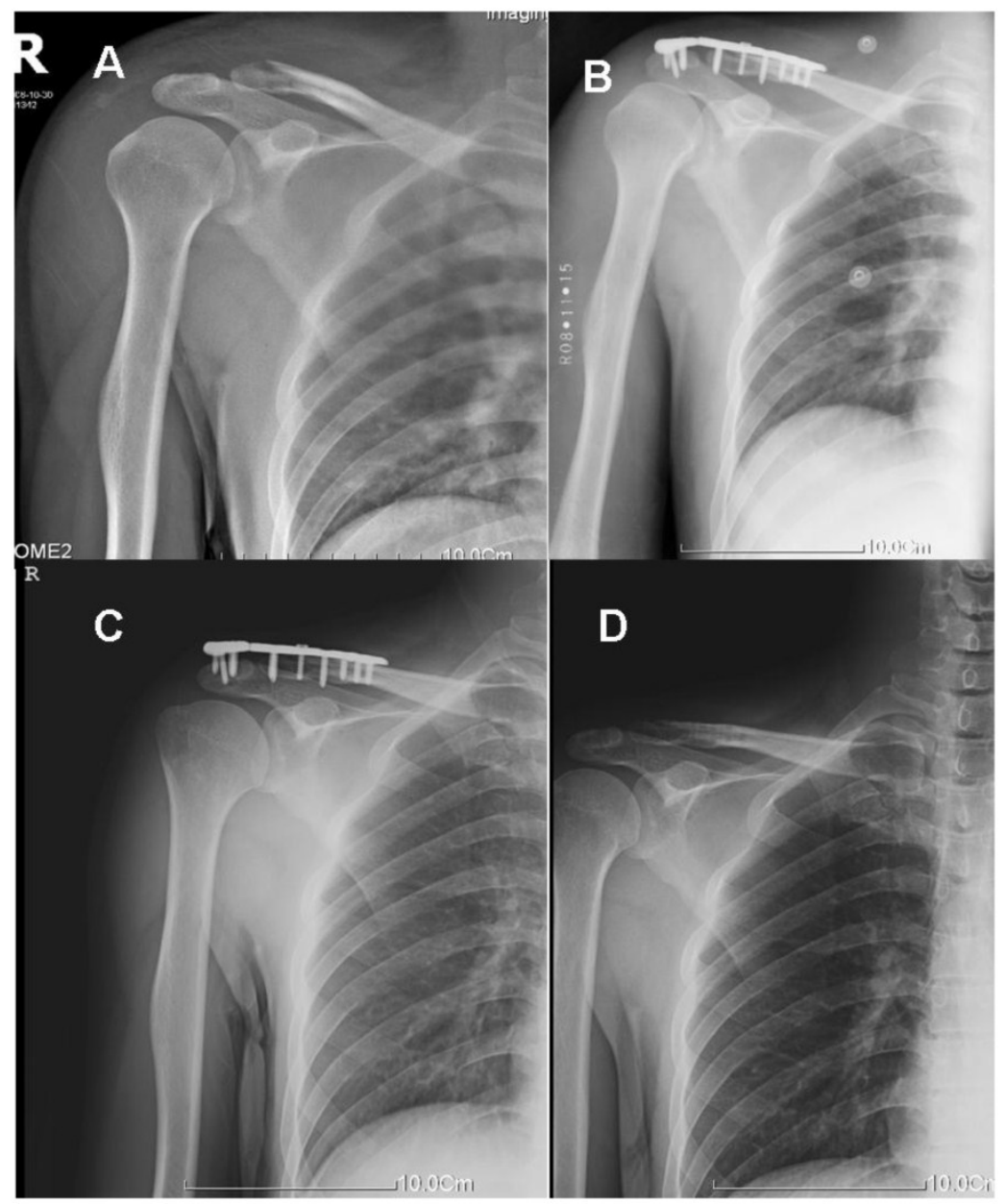

Figure 2 Shoulder anteroposterior radiograph of a 33-year-old man with distal clavicle fracture. (A) Preoperative radiograph showing Neer type llb distal clavicle fracture with mild displacement of the proximal clavicle. (B) Postoperative one week, a radiograph showed the Neer type IIB distal clavicle fracture was fixed with the MAAP fixation technique. (C) Three months after surgery, a radiograph showed fracture healing with ideal reduction of the distal clavicle. (D) One year after surgery, the MAAP was removed without any redisplacement. 
Table 2. Patient demographics, functional outcome, healing time, and complications.

\begin{tabular}{|c|c|c|c|c|c|c|c|}
\hline Case & Age(years) & Sex & Side & Neer classification & Karlsson's criteria & Complications & Time to union (weeks) \\
\hline 1 & 19 & $\mathrm{M}$ & Left & Type IIA & A & None & 8 \\
\hline 2 & 20 & $\mathrm{~F}$ & Left & Type IIA & A & None & 10 \\
\hline 3 & 18 & M & Left & Type IIB & A & None & 12 \\
\hline 4 & 33 & $\mathrm{~F}$ & Right & Type IIA & B & None & 9 \\
\hline 5 & 34 & M & Right & Type IIB & A & None & 12 \\
\hline 6 & 33 & $\mathrm{~F}$ & Right & Type IIA & A & None & 12 \\
\hline 7 & 35 & $\mathrm{M}$ & Right & Type IIB & B & None & 13 \\
\hline 8 & 40 & M & Left & Type IIB & A & None & 14 \\
\hline 9 & 32 & M & Right & Type IIA & A & None & 16 \\
\hline 10 & 19 & $\mathrm{M}$ & Left & Type IIB & A & Nonunion & 13 \\
\hline 11 & 20 & $\mathrm{~F}$ & Right & Type IIB & A & None & 15 \\
\hline 12 & 41 & $\mathrm{~F}$ & Left & Type IIA & A & None & 10 \\
\hline 13 & 44 & $\mathrm{M}$ & Right & Type IIB & A & None & 10 \\
\hline 14 & 34 & $\mathrm{~F}$ & Right & Type IIA & B & None & 12 \\
\hline 15 & 35 & $\mathrm{~F}$ & Right & Type IIB & B & None & 16 \\
\hline 16 & 44 & $\mathrm{M}$ & Right & Type IIB & A & None & 11 \\
\hline 17 & 32 & $\mathrm{M}$ & Right & Type IIB & A & None & 12 \\
\hline 18 & 43 & $\mathrm{~F}$ & Right & Type IIB & A & None & 11 \\
\hline
\end{tabular}

\section{Discussion}

Fractures of the distal clavicle are usually unstable comparing with proximal clavicle fractures, especially type II fractures are often displaced and may have a higher rate of nonunion ${ }^{[7]}$. According to the relevant literature statistics, rates of nonunion following nonsurgical treatment of type II distal clavicle fractures range from $28 \%$ to $44 \%$, and surgical treatment is generally recommended $\left[{ }^{8,9]}\right.$. There are many choices of surgical treatment of this type of fracture, including k-wire fixation, clavicular hook plate fixation, modified tension band fixation, and bosworth-type screw fixation, which also have frequent complications as a result of either fixation itself or fixing together with acromioclavicular joint limiting its micro-movable feature ${ }^{[9-14]}$, and there is no consensus regarding the most reliable treatment for providing the most effective clinical outcomes.

The acromioclavicular joint is a plane diathroidal joint formed from the outer surfaces of the lateral end of the clavicle and the anterior aspect of the acromion, and contains a fibrocartilaginous disc ${ }^{[15]}$. The range of movement at the acromioclavicular joint has been investigated for many years. In the early cadaveric study, Moseley et al.[16] reported that the acromioclavicular joint had a certain degree of rotation. Recently, three-dimensional studies of the acromioclavicular joint using open MR imaging showed that the acromioclavicular joint has approximately $15-20^{\circ}$ of rotation ${ }^{[17]}$, which support the view that the acromiocla- vicular joint has some degree of rotational movements. Therefore, combining with clinical considerations, the operative methods for treating distal clavicle fracture through rapid fixation between the clavicle and acromion or coracoid, that is, the fixations such as k-wire, clavicular hook plate, and coracoclavicular screw will limit the postoperative movement of the acromioclavicular joint to some extent, which are thus inadvisable for the treatment of distal clavicular fractures.

The purpose of surgery is to maintain the stability of the acromioclavicular joint and promote early recovery of shoulder function. In all type II fractures, the proximal fragment is detached from the coracoclavicular ligaments. Therefore, reconstruction of coracoclavicular ligament is very important for type II distal clavicle fractures, which can provide superior primary stability for the fixation of distal clavicle. If the ruptured coracoclavicular ligament does not heal, the fixation methods may ultimately lead to failure. So, for all type II fractures, the coracoclavicular ligament should be performed routinely to repair.

The MAAP is designed according to the superior surface morphology of the distal clavicle and the acromial end, include oval acromial end and relatively flat, thin, wide of the clavicle end. The plate can attach to the superior part of the distal clavicle and the acromial more closely without bending, and anatomical reduction of fractures can be firmly maintained without damaging the acromioclavicular joint capsule and ligaments. Moreover, the MAAP contains a 
three-dimensional motion connecting rods, so internal fixation using this plate will not interfere with the extra-articular or intra-articular motion postoperatively. The arm is kept in a forearm sling for 2 weeks in all cases during the acute painful period after surgery, and a patient's usual daily activities are permitted relatively early after MAAP fixation.

In this series of patients, the use of MAAP fixation can both effectively fix the unstable distal clavicle fractures and do not affect the motion of the acromioclavicular joint, while maintaining integrity of the acromioclavicular joint capsule. On the other hand, the MAAP is a limited contact plate, which can minimize the impact of the blood supply to the periosteum and cortex and would not devascularize the clavicle. Therefore, the technique described in this study fulfills the principles of biological fixation and early functional exercises. Although general anesthesia is required for the internal fixation removal, the MAAP fixation method seems to be a suitable option for treating unstable distal clavicle fractures, especially those involving comminuted fractures or osteoporotic fractures.

In this study, all the patients were taken early passive shoulder joint exercises 1 week after surgery, and early functional recovery was observed. An excellent and encouraging functional result was observed as early as 3 months postoperatively. Some of the complications that may arise such as loosening, migration, breakage have not been observed.

In conclusion, the use of MAAP of fixation of Neer II distal clavicle fractures can provide stable fixation with a low rate of complications and achieve satisfactory functional outcome. We believe that it is one of good option for treating unstable distal clavicle fractures. However, it is necessary to continue to observe the clinical effects of this fixation method.

\section{Acknowledgement}

This work was supported by "Eleventh Five-Year" scientific research Project of Nanjing Military Region; contract grant number: 06NA100.

\section{Competing Interests}

The authors have declared that no competing interest exists.

\section{References}

[1] Ballmer FT, Gerber C. Coracoclavicular screw fixation for unstable fractures of the distal clavicle. A report of five cases. J Bone Joint Surg Br. 1991. 73(2): 291-4

[2] 2nd NCS. Fractures of the distal third of the clavicle. Clin Orthop Relat Res. 1968. 58: 43-50.

[3] 2nd NCS. Fracture of the distal clavicle with detachment of the coracoclavicular ligamentsin adults. J Trauma. 1963. 3: 99-110.

[4] 2nd NCS. Nonunion of the clavicle. J Am Med Assoc. 1960. 172: 1006-11.
[5] Edwards DJ, Kavanagh TG, Flannery MC. Fractures of the distal clavicle: a case for fixation. Injury. 1992. 23(1): 44-6.

[6] Li Y, Shi S, Ou-Yang YP, Liu TL. Minimally invasive treatment for Neer IIb distal clavicle fractures with titaniumcable. J Trauma. 2011. 71(2): E37-40.

[7] 2nd NCS. Fracture of the distal clavicle with detachment of the coracoclavicular ligamentsin adults. J Trauma. 1963. 3: 99-110.

[8] Banerjee R, Waterman B, Padalecki J, Robertson W. Management of distal clavicle fractures. J Am Acad Orthop Surg. 2011. 19(7): 392-401.

[9] Kona J, Bosse MJ, Staeheli JW, Rosseau RL. Type II distal clavicle fractures: a retrospective review of surgical treatment. J Orthop Trauma. 1990. 4(2): 115-20.

[10] Di FA, Zoccali C, Colafarina O, Pizzoferrato R, Flamini S. The use of hook plate in type III and $\mathrm{V}$ acromio-clavicular Rockwood dislocations: Clinical and radiological midterm results and MRI evaluation in 42 patients. Injury. 2012. 43(2): 147-52.

[11] Chun JM, Kim SY. Modified tension band fixation for unstable distal clavicle fractures. J Trauma. 2011. 70(5): E88-92.

[12] Kashii M, Inui H, Yamamoto K. Surgical treatment of distal clavicle fractures using the clavicular hook plate. Clin Orthop Relat Res. 2006. 447: 158-64.

[13] Flinkkila T, Ristiniemi J, Hyvonen P, Hamalainen M. Surgical treatment of unstable fractures of the distal clavicle: a comparative study of Kirschner wire and clavicular hook plate fixation. Acta Orthop Scand. 2002. 73(1): 50-3.

[14] Yamaguchi H, Arakawa H, Kobayashi M. Results of the Bosworth method for unstable fractures of the distal clavicle. Int Orthop. 1998. 22(6): 366-8.

[15] Colegate-Stone T, Allom R, Singh R, Elias DA, Standring S, Sinha J. Classification of the morphology of the acromioclavicular joint using cadavericand radiological analysis. J Bone Joint Surg Br. 2010. 92(5): 743-6.

[16] Moseley HF. The clavicle: its anatomy and function. Clin Orthop Relat Res. 1968. 58: 17-27.

[17] Sahara W, Sugamoto K, Murai M, Yoshikawa H. Three-dimensional clavicular and acromioclavicular rotations during arm abduction using vertically open MRI. J Orthop Res. 2007. 25(9): 1243-9. 